\title{
Microbiology of chili bo, a popular Malaysian food ingredient
}

\begin{abstract}
The predominant microbial flora of a specific Malaysian food ingredient. chili bo (containing $9 \%$ ground dried chilies. $0.6 \%$ acetic acid, and 5 to $10 \%$ cornstarch, wt/vol) stored for up to 25 days at $28^{\circ} \mathrm{C}$ without added benzoic acid (product A) and with 7,000 ppm of added benzoic acid (product B) was examined. Aerobic plate counts for both products were initially 6.2 to $6.5 \log \mathrm{CFU} / \mathrm{g}$ increasing to $8.5 \log \mathrm{CFU} / \mathrm{g}$ for product A after 4 days. Aerobic plate counts for product $B$ did not increase during storage. Lactic acid bacteria (LAB) counts increased in product A from $4.8 \log \mathrm{CFU} / \mathrm{g}$ to $8.3 \mathrm{log} \mathrm{CFU} / \mathrm{g}$ and in product B from $2.1 \mathrm{log}$ $\mathrm{CFU} / \mathrm{g}$ to $7.6 \log \mathrm{CFU} / \mathrm{g}$ after 17 days. Growth of yeast occurred in product A. Both products exhibited spoilage after 1 to 2 days of storage at $28^{\circ} \mathrm{C}$ indicated as accumulation of gas bubbles. In addition surface growth of molds (product A) or whitish discoloration (product B) was observed later in storage. For product A the predominant isolates were LAB. Bacillus pumilus, Bacillus subtilis, Staphylococcus spp., and yeasts. B. pumilus and B. subtilis predominated initially whereas the other types of microorganisms predominated after 25 days of storage. B. pumilus and B. subtilis were also predominant in product B, but after 25 days of storage a homofermentative LAB was found in higher numbers (7.6 log CFU/g). Isolates of heterofermentative LAB but not homofermentative LAB or B. pumilus or B. subtilis were able to produce gas during growth in chili be sterilized by autoclaving at $121^{\circ} \mathrm{C}$ for 15 min. Growth of heterofermentative LAB, B. pumilus, and B. subtilis was inhibited by acidifying agents, a nisin-containing supernatant, or incubation at low temperatures.
\end{abstract}

Keyword: Bacillus; Benzoic acid; Chili bo; Inhibition; Lactic acid bacteria; Nisin; Spoilage 